\title{
Colagenosis perforante reactiva adquirida
}

\section{Acquired reactive perforating collagenosis}

\author{
José Alberto Ramos-Garibay, ${ }^{*}$ Juan Ramón Trejo-Acuña, ${ }^{\ddagger}$ Nayeli Meza-Gaxiola§
}

\section{RESUMEN}

Las dermatosis perforantes constituyen un grupo de enfermedades que se caracterizan clínicamente por la eliminación de diversos materiales cutáneos por vía transepidérmica. Se han descrito cuatro formas clásicas primarias en las que el material de eliminación representa el cuadro clínico distintivo. En las secundarias, la perforación y eliminación de las fibras del tejido conjuntivo constituyen un fenómeno que acompaña a otras dermatosis. La colagenosis perforante reactiva adquirida pertenece a este último grupo. La etiología no es clara, se le asocia con trastornos metabólicos y ciertas neoplasias. El prurito se considera un factor patogénico clave, ya que el rascado constante genera microtraumatismos en pacientes genéticamente predispuestos, provocando la degeneración focal de las fibras de colágeno. La topografía más frecuente es en el tronco $(77 \%)$, seguido de las extremidades $(64 \%)$, de las que compromete las zonas extensoras. Se manifiesta clínicamente con la presencia de pápulas eritematosas de aspecto umbilicado, con un tapón de queratina firmemente adherido en el centro. En la histopatología se observa eliminación transepidérmica de fibras de colágeno. Debido a que el tratamiento no está aún bien estandarizado, el manejo del prurito se considera una de las bases del mismo. Se recomienda tratar las enfermedades asociadas.

Palabras clave: Dermatosis perforantes, colagenosis perforante reactiva adquirida, enfermedades por eliminación transepidérmica.

\begin{abstract}
Perforating dermatoses are a group of diseases clinically characterized by transepidermal shedding of various skin materials. Four primary classical forms have been described in which the removal material represents the distinctive clinical picture. In secondary cases, the perforation and elimination of the fibers of the connective tissue constitute a phenomenon that accompanies other dermatoses. Acquired reactive perforating collagenosis belongs to the latter group. The etiology is not clear. It is associated with metabolic disorders and certain neoplasms. Pruritus is considered a key pathogenic factor, since constant scratching generates microtrauma in genetically predisposed patients, causing focal degeneration of collagen fibers. The most frequent topography is in the trunk (77\%) and the extremities (64\%), of which the extensor areas are involved. It manifests clinically with the presence of erythematous papules with an umbilicus appearance, with a firmly adhered keratin plug in the center. Histopathology shows transepidermal removal of collagen fibers. Because the treatment is not yet well standardized, the management of pruritus is considered one of the bases of it. It is recommended to treat the associated diseases.
\end{abstract}

Keywords: Perforating dermatoses, acquired reactive perforating collagenosis, transepidermal elimination diseases.

\section{INTRODUCCIÓN}

Las enfermedades por eliminación transepidérmica (también llamadas dermatosis perforantes) constituyen un grupo heterogéneo de enfermedades de la piel definido por la eliminación transepidérmica de diversos materiales cutáneos. Se han descrito cuatro formas clásicas de dermatosis perforante primaria, donde el mecanismo de eliminación transepidérmica representa el sello distintivo de la enfermedad: colagenosis perforante

\footnotetext{
* Dermatopatólogo.

‡ Dermatólogo y Dermatooncólogo.

$\S$ Residente del segundo año de Dermatología.

Centro Dermatológico «Dr. Ladislao de la Pascua», SSCDMX. México.
}

Citar como: Ramos-Garibay JA, Trejo-Acuña JR, Meza-Gaxiola N. Colagenosis perforante reactiva adquirida. Rev Cent Dermatol Pascua. 2021; 30 (3): 141-147. https://dx.doi. org/10.35366/103828 
reactiva adquirida, elastosis perforante serpiginosa, enfermedad de Kyrle y foliculitis perforante..$^{1,2}$

Rapini y colaboradores en 1989 le denominaron dermatosis perforante adquirida. ${ }^{3}$ En la actualidad, dado que existen casos de la enfermedad de inicio temprano, que no necesariamente se pueden catalogar como adquiridos, se les engloba colectivamente como dermatosis perforantes.

Cada entidad fue reportada por primera vez por los siguientes autores: enfermedad de Kyrle en 1916 por Kyrle, ${ }^{4}$ elastosis perforante serpiginosa en 1953 por Lutz, ${ }^{5}$ foliculitis perforante y colagenosis perforante reactiva adquirida en 1968 por Mehregan y Coskey (Tabla 1). ${ }^{6}$

Kawakami y colaboradores ${ }^{1}$ propusieron que debido a que las sustancias que se eliminan por vía transepidérmica no están aún claramente clasificadas para cada entidad, se agregara el término «principalmente», ya que en un mismo paciente se puede observar la eliminación de varios elementos de la dermis.

En las dermatosis perforantes secundarias, la perforación y eliminación de las fibras del tejido conjuntivo es un fenómeno ocasional, que ocurre acompañando a otras dermatosis, tales como: granuloma anular, calcinosis cutis, necrobiosis lipoídica, condrodermatitis nodular del hélix crónico, queratoacantoma, entre otras. ${ }^{2}$

Tabla 1: Criterios diagnósticos para dermatosis perforantes.
A
Hallazgos básicos
Los hallazgos histopatológicos muestran eliminación transepidérmica de componentes cutáneos degenerados
B Sustancias que se eliminan principalmente por vía transepidérmica
1 Fibras de colágeno
2 Fibras elásticas
3 Queratinas
4 Tejido colágeno propio de los folículos pilosos
C Hallazgos clínicos cutáneos
1 Pápulas o lesiones de aspecto nodular y umbilica- do con tapón queratósico adherente central
2 Inicio a los 18 años o más
D Hallazgos de referencia
Fenómeno de Koebner
Prurito

Categoría de diagnóstico (se realiza un diagnóstico definitivo en los siguientes casos):

$\mathrm{A}+\mathrm{B} 1+\mathrm{C} 1+\mathrm{C} 2=$ colagenosis perforante reactiva adquirida

$A+B 2=$ elastosis perforante serpiginosa

$A+B 3=$ enfermedad de Kyrle

$A+B 4=$ foliculitis perforante

Tomado de: Kawakami T et al.
La colagenosis perforante reactiva adquirida (CPRA) se caracteriza por la eliminación transepidérmica de las fibras de colágeno, en la elastosis perforante serpiginosa, las fibras elásticas son las que se eliminan, en la enfermedad de Kyrle la queratina anormal, y en la foliculitis perforante el contenido del folículo. ${ }^{1}$

\section{Epidemiología}

Debido a su baja frecuencia, se desconocen la prevalencia e incidencia exactas de esta dermatosis. Shakery y Saray con sus respectivos colaboradores ${ }^{7,8}$ reportaron frecuencias similares: 10 casos en un periodo de cinco años. Karpouzis y colaboradores realizaron una revisión de la literatura de 101 pacientes y encontraron discreto predominio en hombres: 1.5:1. La edad de presentación al momento del diagnóstico osciló entre los 29 y los 96 años, con una media de 56.8 años. $^{7-9}$

La CPRA se manifiesta en adultos, diferenciándose de la forma hereditaria, la cual se desarrolla en niños y adolescentes. ${ }^{2}$

\section{Etiología}

La etiología y patogenia de este padecimiento aún no están bien establecidas; sin embargo, se le asocia con trastornos metabólicos (diabetes mellitus, enfermedad renal crónica, hiperuricemia) y a procesos neoplásicos. El prurito crónico se considera un factor patogénico clave, ya que provoca microtraumatismos que en pacientes genéticamente predispuestos puede ser el responsable de la degeneración focal de las fibras de colágeno. 2,10 La relación patogénica entre el prurito y la aparición de las lesiones características se sustenta en la ubicación de las mismas, ya que generalmente están al alcance de las manos, así como en la presencia del fenómeno de Koebner, y en el hecho de observar mejoría de los síntomas con un tratamiento antipruriginoso. De igual forma, los estudios de inmunohistoquímica han demostrado la activación e incremento de los factores de crecimiento y de las enzimas de la matriz extracelular, tales como el factor de crecimiento transformante beta 3 (TGF- $\beta 3$, por sus siglas en inglés), así como aumento en la actividad de los fibroblastos a nivel intralesional. ${ }^{10,11}$

\section{Cuadro clínico}

Los sitios más afectados son el tronco (77\%), la cintura escapular, los glúteos y extremidades superiores e inferiores (64\%), predomina en áreas extensoras (zonas 
Tabla 2: Clasificación de la gravedad de las dermatosis perforantes.

1

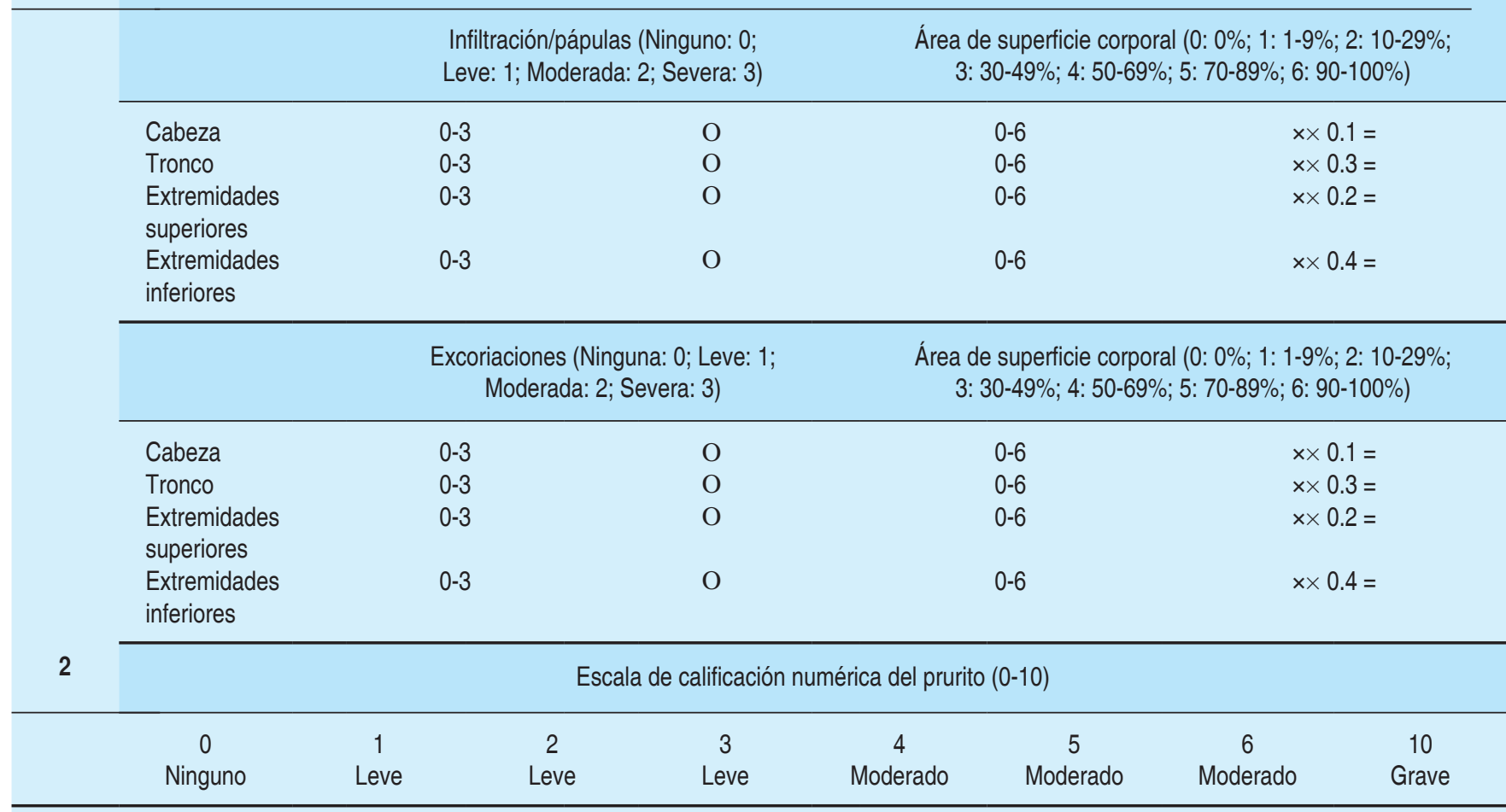

$1+2$ = valor de puntuación total (puntos decimales redondeados)

Casi remisión: 0-1. Leve: 2-6. Moderado: 7 a 15. Grave: 16-33. Más grave: 34-46

Tomado de: Kawakami T et al. ${ }^{1}$

más accesibles al rascado), y la cabeza (22\%). Las regiones palmoplantares, las áreas intertriginosas y las membranas mucosas no están involucradas. .,7,9 $^{2,9}$

En 1968 Mehregan y Coskey describieron casos de CPRA con múltiples pápulas y placas eritematosas de aspecto umbilicado, con un tapón queratósico adherente en el centro; en ocasiones también podían observarse costras color marrón verdoso o negro, firmemente adheridas. ${ }^{2,6}$ Las lesiones pueden medir hasta $2 \mathrm{~cm}$, algunas confluyen formando placas de hasta $8 \mathrm{~cm}$ de diámetro. ${ }^{7,10,12}$

Kim y colaboradore ${ }^{13}$ estudiaron 30 casos de CPRA y detectaron la presencia de prurito en $83.3 \%$ de los pacientes, así como fenómeno de Koebner en 31.8\%, signos y síntomas útiles para realizar el diagnóstico.

Kawakami y colaboradores ${ }^{1}$ clasificaron la gravedad de las dermatosis perforantes basándose en el Eczema Area and Severity Index (EASI) empleado para la dermatitis atópica, publicada en 2001 por Hanifin y colaboradores. ${ }^{14}$ Tomaron como elemento 1 la presencia de infiltración y pápulas, y como elemento 2 el prurito. Al sumar el elemento 1 y el 2 se obtuvo un puntaje, el cual se calificó de la siguiente manera: (I) casi remisión (0-1 punto), (II) leve (2-6 puntos), (III) moderado (7 a 15 puntos), (IV) grave (16-33 puntos), (V) más grave (34-46 puntos) (Tabla 2).

\section{Comorbilidades}

Se ha observado que la CPRA se presenta en asociación con diabetes mellitus, enfermedad renal crónica, hiperuricemia y en algunas ocasiones con neoplasias (hay autores que consideran esta dermatosis como un trastorno paraneoplásico). ${ }^{1,2}$ Existen otros padecimientos menos comunes, tales como hipotiroidismo, enfermedad de Hodgkin, carcinoma hepatocelular, cáncer de tiroides y dermatomiositis, entre otros. , $^{2,15}$

De igual forma, existen reportes de su presentación después de una picadura de insecto, de un cuadro de escabiosis o de herpes zóster. ${ }^{16,17}$ 
Se le ha relacionado también con la ingesta de algunos medicamentos, entre ellos clopidogrel, indinavir y sirolimus. ${ }^{18-20}$

\section{Diagnóstico}

En cuanto a la CPRA, Faver y colaboradores publicaron que los criterios para el diagnóstico son los siguientes: (I) hallazgos histopatológicos de eliminación de tejido colágeno necrótico a través de la epidermis; (II) presencia de pápulas de aspecto umbilicado, o lesiones de aspecto nodular con tapón queratósico adherente en el centro y (III) aparición de lesiones cutáneas después de los 18 años.

Los diagnósticos diferenciales incluyen, entre otros: pitiriasis liquenoide crónica y prurigo nodular. ${ }^{1,8,9}$

\section{Histopatología}

Estas enfermedades se caracterizan por el hallazgo de eliminación transepidérmica de componentes desnaturalizados o degenerados de la dermis (fibras de colágeno) ${ }^{1}$

Se ha demostrado la eliminación transepidérmica del colágeno tipo IV. Se observa deformidad dérmica en forma de copa que contiene colágeno basófilo degenerado, el cual caracteriza a las lesiones tempranas. En ocasiones la epidermis se observa atrófica, en el centro presenta una capa delgada de hiperquerato- sis con paraqueratosis. En los márgenes laterales, típicamente hay acantosis. En la lesión umbilicada, completamente establecida, el tapón central está compuesto por detritos paraqueratósicos, colágeno degenerado y células inflamatorias. La epidermis está marcadamente atrófica y atravesada focalmente por fibras de colágeno orientadas verticalmente. Las fibras elásticas no están presentes dentro de los restos de tejido conectivo extruidos. A ambos lados de la deformidad en forma de copa, la epidermis es acantótica e hiperqueratósica. Hay infiltrado linfohistiocítico en la dermis superficial. En la colagenosis perforante reactiva y la colagenosis perforante adquirida el aspecto histológico es idéntico. ${ }^{21}$

\section{Tratamiento}

Actualmente no existen ensayos clínicos que evalúen y comparen la respuesta al tratamiento. La información existente a este respecto se basa en el reporte de casos. El tratamiento sintomático del prurito se considera uno de los pilares en el manejo. ${ }^{7}$ Este incluye formulaciones con urea y propilenglicol. Para el tratamiento sistémico, se pueden administrar antihistamínicos y, si es necesario, en combinación con otros agentes, como esteroides.

La fototerapia es otra opción para tratar el prurito (por sus efectos antiinflamatorios y antiproliferativos directos), generalmente ultravioleta B (UVB). ${ }^{22-24}$
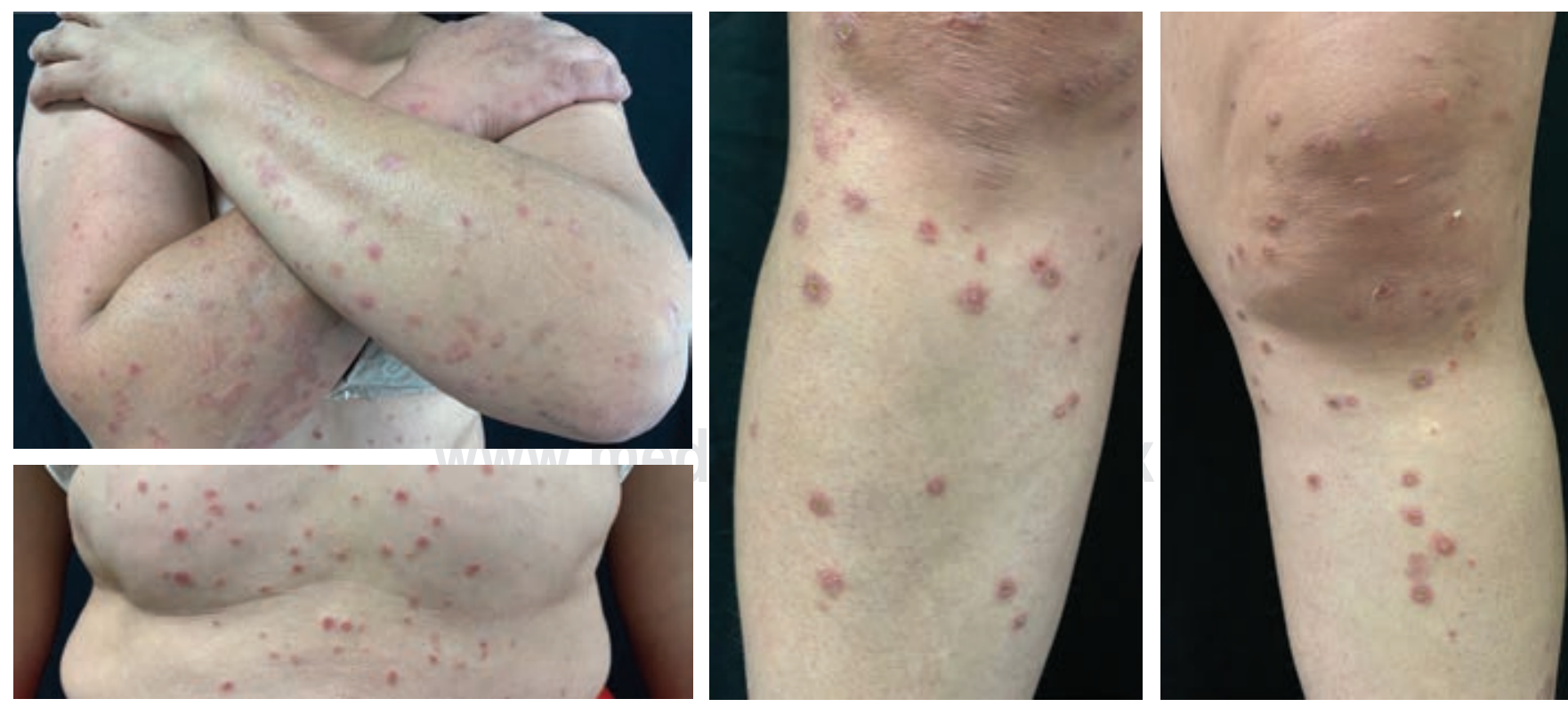

Figura 1: Placas eritematosas con escama queratósica en el centro, algunas milimétricas, muy pruriginosas. 
Figura 2: Se observa material necrótico con fibras colágenas degeneradas protruyendo a través de la epidermis (H\&E 4x).

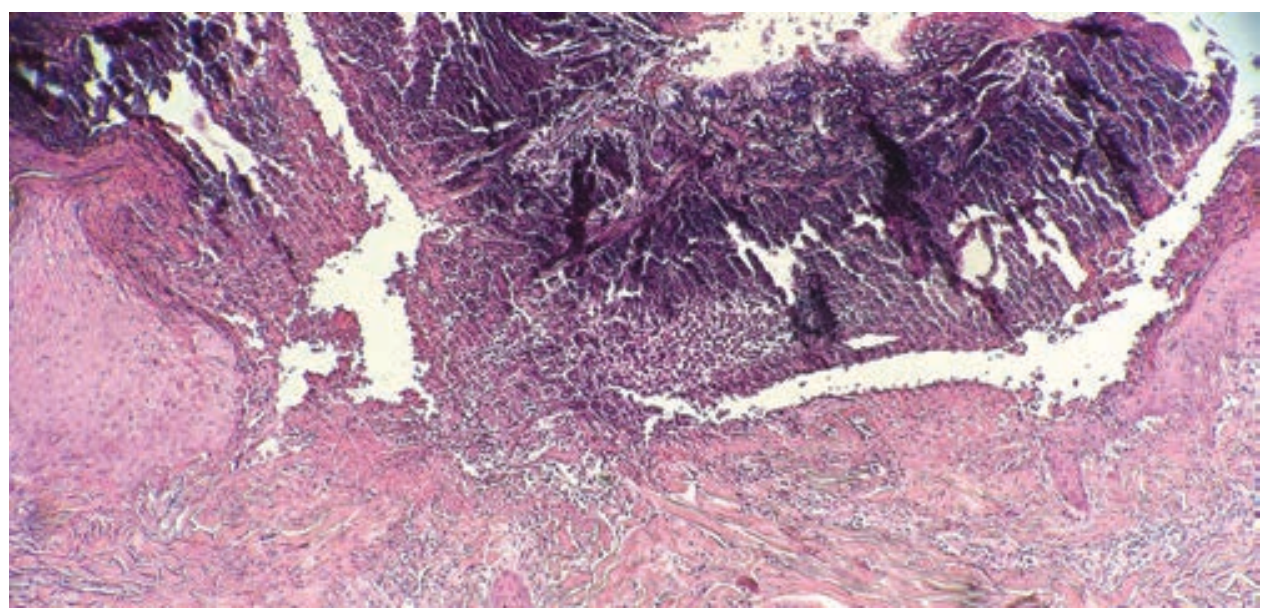

Las formas localizadas se tratan inicialmente con la aplicación de agentes queratolíticos como el ácido salicílico en concentración de 5 a $7 \%$, urea de 10 a $15 \%$ y tretinoína de 0.01 a $0.1 \%$; si es necesario, pueden aplicarse bajo oclusión. 2,7,23,25

En cuanto al tratamiento antiinflamatorio, se pueden emplear corticosteroides tópicos de clase II-III, aplicación intralesional de triamcinolona, al igual que retinoides tópicos. Para el tratamiento sistémico se pueden utilizar corticosteroides y retinoides, algunos autores recomiendan alopurinol; sin embargo, los resultados son contradictorios.

Se han reportado casos aislados de tratamiento con roxitromicina, doxiciclina, hidroxicloroquina, sulfona (dapsona) y metotrexato.

Se recomienda el tratamiento de las enfermedades de base. No existe evidencia que lo respalde; no obstante, en algunas series de casos, el tratamiento del padecimiento de base, como la diabetes mellitus y la enfermedad renal crónica demostraron ser eficaces para la mejoría de la dermatosis. ${ }^{1,2,25,26}$

Al momento de esta publicación el pronóstico se desconoce, ya que no existe evidencia suficiente para determinar la evolución de los pacientes, así como el porcentaje de recuperación o recurrencias, debido al poco número de casos publicados.

\section{CASO CLÍNICO}

Mujer de 42 años de edad, originaria y residente del Estado de México, de ocupación comerciante. Acude por presentar una dermatosis diseminada a tronco, extremidades superiores e inferiores, bilateral y asimétrica. Afecta tórax anterior, abdomen, tórax posterior y región lumbar; también afecta brazos, antebrazos, manos, muslos, piernas y pies en todas sus caras. Constituida

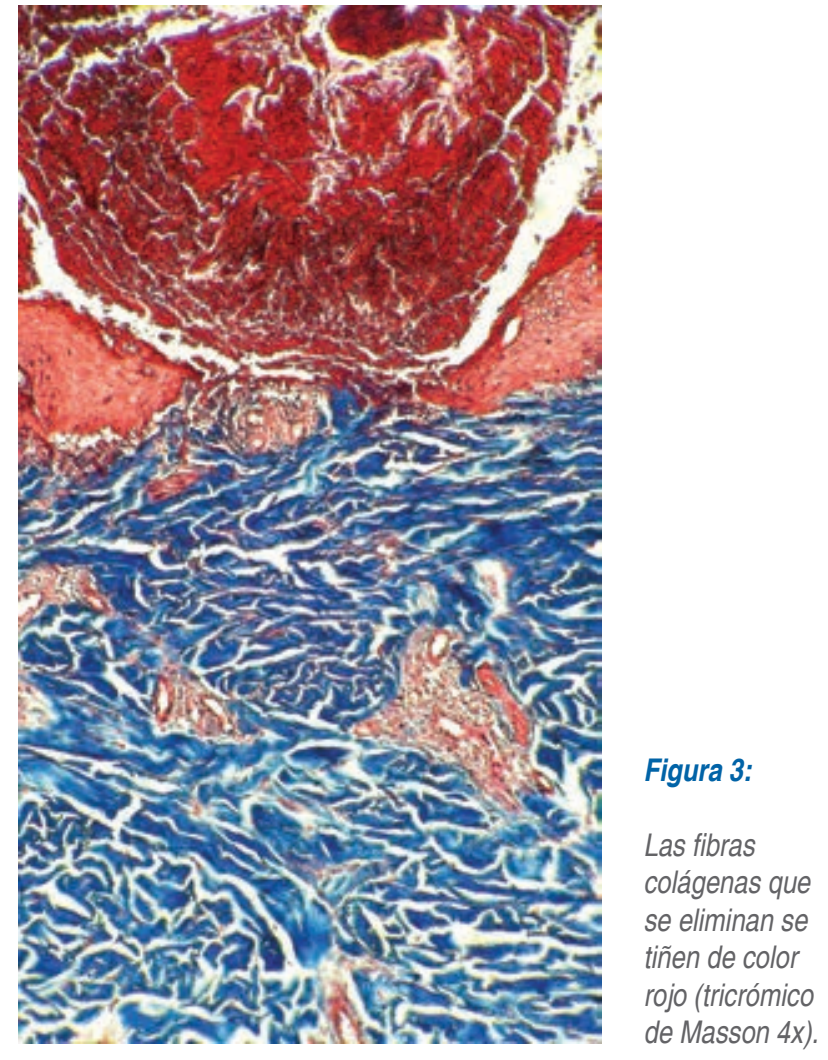

por numerosas placas eritematosas, de forma y tamaño variable, algunas milimétricas, de bordes irregulares, mal definidos, y superficie con fina escama laminar en el centro, adherente, algunas con exulceración y costras hemáticas (Figura 1). De evolución crónica y pruriginosa. Refiere haber iniciado el padecimiento actual 20 días antes, con las lesiones ya señaladas, acompañadas de prurito en aumento. Le fueron administrados múltiples 
tratamientos previos, tales como betametasona, clotrimazol y gentamicina tópica por 15 días, cefalexina cada 8 horas por dos días, ivermectina 12 mg cada 24 horas, dexametasona $8 \mathrm{mg}$ intramuscular en dosis única, ibuprofeno tópico, prednisona $75 \mathrm{mg}$ cada 24 horas por ocho días, levocetirizina $5 \mathrm{mg}$ cada 12 horas, loratadina $10 \mathrm{mg}$ cada 24 horas. Sin antecedentes personales patológicos de importancia para su padecimiento actual.

Se realizó biopsia incisional, la cual reportó epidermis con acantosis e hiperqueratosis, así como zona central con ausencia de epidermis sustituida por restos celulares y fibras colágenas degeneradas provenientes de la dermis. Se realizó el diagnóstico histopatológico de enfermedad por eliminación transepidérmica (Figuras 2 y 3). Por correlación clínico-patológica, se estableció el diagnóstico de colagenosis perforante reactiva. Por su alta asociación con enfermedades metabólicas y neoplasias, solicitamos exámenes complementarios, con los cuales se descartaron dichas entidades. Se manejó con emoliente (cold cream) y antihistamínico (levocetirizina $10 \mathrm{mg}$ al día). Se hizo énfasis en la aplicación constante del primero y en la toma con horario del segundo. Actualmente con buena evolución, con disminución progresiva de la dermatosis. Continúa en seguimiento por nuestro servicio.

\section{COMENTARIO}

Las dermatosis perforantes se consideran poco frecuentes. La CPRA se asocia frecuentemente con enfermedades metabólicas y neoplasias. La histopatología es esencial para corroborar el diagnóstico; en ésta se observa la característica eliminación transepidérmica de fibras de colágeno.

Hasta el momento no existen ensayos clínicos que reporten el tratamiento más adecuado para esta entidad.

El interés de publicar este caso radica en que se trata de un cuadro idiopático, lo cual es poco común, ya que no se encontró patología agregada que lo condicionara. De gran utilidad fue la realización de una biopsia incisional, con lo cual se corroboró el diagnóstico.

Por otro lado, la mejoría observada se obtuvo tras la explicación detallada y el adecuado apego al tratamiento antipruriginoso por parte de la paciente.

Al momento de esta publicación, la paciente no ha presentado recurrencia.

\section{REFERENCIAS}

1. Kawakami T, Akiyama M, Ishida-Yamamoto A, Nakano H, Mitoma C, Yoneda $\mathrm{K}$ et al. Clinical practice guide for the treatment of perforating dermatosis. J Dermatol. 2020; 47: 1374-1382. doi: 10.1111/13468138.15647.

2. Wagner G, Sachse MM. Acquired reactive perforating dermatosis. $J$ Dtsch Dermatol Ges. 2013; 11: 723-729. doi: 10.1111/ddg.12131.

3. Rapini RP, Herbert AA, Drucker CR. Acquired perforating dermatosis. Evidence for combined transepidermal elimination of both collagen and elastic fibers. Arch Dermatol. 1989; 125: 1074-1078.

4. Kyrle J. Hyperkeratosis follicularis et parafollicularis in cutem penetrans. Arch Dermatol Syphil, 1916; 123: 466-493.

5. Lutz W. Keratosis follicularis serpiginosa. Dermatologica. 1953; 106: 318-319.

6. Mehregan AH, Coskey RJ. Perforating folliculitis. Arch Dermatol. 1968; 97: 394-399.

7. Shakery K, Zimmermann J, Bahmer FA. Erworbene reaktiv perforierende dermatose im patientengut der Dermatologischen Klinik Bremen 2003-2006 [in German]. Akt Dermatol. 2008; 34: 409-413.

8. Saray $Y$, Seckin D, Bilezikci B. Acquired perforating dermatosis: clinicopathological features in twenty-two cases. J Eur Acad Dermatol Venereol. 2006; 20: 679-688.

9. Karpouzis A, Giatromanolaki A, Sivridis E, Kouskoukis C. Acquired reactive perforating collagenosis: current status. J Dermatol. 2010; 37: 585-592.

10. Faver IR, Daoud MS, Su WP. Acquired reactive perforating collagenosis. Report of six cases and review of the literature. J Am Acad Dermatol. 1994; 30: 575-580.

11. Poliak SC, Lebwohl MG, Parris A, Prioleau PG. Reactive perforating collagenosis associated with diabetes mellitus. N Engl J Med. 1982; 306: 81-84.

12. Matthes T, Trost TH, Nilles M. Erworbene reaktive perforierende Dermatose bei Psoriasis vulgaris. Akt Dermatol. 2001; 27: 74-78.

13. Kim SW, Kim MS, Lee JH, Son SJ, Park KY, Li K et al. A clinicopathologic study of thirty cases of acquired perforating dermatosis in Korea. Ann Dermatol. 2014; 26: 162-171.

14. Hanifin JM, Thurston M, Omoto M, Cherill R, Tofte SJ, Graeber M. The eczema area and severity index (EASI): assessment of reliability in atopic dermatitis. EASI Evaluator Group. Exp Dermatol. 2001; 10: 11-18.

15. Yazdi S, Saadat P, Young S, Hamidi R, Vadmal MS. Acquired reactive perforating collagenosis associated with papillary thyroid carcinoma: a paraneoplastic phenomenon? Clin Exp Dermatol. 2010; 35: 152-155.

16. Kim EJ, Kim MY, Kim HO, Park YM. Acquired reactive perforating collagenosis triggered by insect bite. J Dermatol. 2007; 34: 677-679.

17. Zanardo L, Stolz W, Landthaler M, Vogt T. Reactive perforating collagenosis after disseminated zoster. Dermatology. 2001; 203: 273-275.

18. Buchau AS, Lewerenz V, Kruse R, Neumann NJ, Ruzicka T, Reinfenberger J. Reaktive perforierende Kollagenose. Hautarzt. 2005; 56: 963-965.

19. Calista D, Morri M. Acquired reactive perforating collagenosis induced by indinavir in 2 patients with HIV disease. Eur J Dermatol. 2008; 18: 84-85.

20. Lübbe J, Sorg O, Malé PJ, Saurat JH, Masouyé I. Sirolimus-induced inflammatory papules with acquired reactive perforating collagenosis. Dermatology. 2008; 216: 239-242.

21. Calonje JE, Brenn T, Lazar AJ, Billings S. McKee's pathology of the skin with clinical correlations. 5th ed. London: Elsevier; 2020. 
22. Scola N, Gambichler T, Altmeyer P, Stucker M, Kreuter A. Erworbene reaktive perforierende Kollagenose nach Herpes zoster als isotopische Antwort? Hautarzt. 2011; 62: 683-687.

23. Schlotmann K, Megahed M, Goerz G. Ungewohnlicher Fall einer erworbenen reaktiven perforierenden Kollagenose. HuG. 1996; 71 : 786-788

24. Cullen SI. Successful treatment of reactive perforating collagenosis with tretinoin. Cutis. 1979; 23: 187-193.

25. Nebel R, Fiedler E, Danz B, Marsch WC, Kreft B. Erworbene reaktiv perforierende Kollagenose bei Diabetes mellitus und dialysepflichtiger Niereninsuffizienz. Dtsch Med Wochenschr. 2007; 132: 2624-2626.
26. Wigger-Alberti W, Richter M, Hochheim B, Schreiber G, Elsner P. Erworbene reaktive perforierende Kollagenose bei Diabetes mellitus. Dtsch Med Wochenschr. 1999; 124: 282-284.

Correspondencia:

Dr. José Alberto Ramos-Garibay

Dr. Vértiz No. 464, esq. Eje 3 Sur,

Col. Buenos Aires, 06780,

Alcaldía Cuauhtémoc, CDMX.

Tel: 55 5519-6351

E-mail: ramosgari4400@yahoo.com.mx 